\title{
Exploration of Proteins Involved in Acquisition of Resistance to Cetuximab
}

\author{
Hironori Nakamura ${ }^{1,2}$, Ayumu Nagamine ${ }^{1,2}$, Hideaki Yashimaa ${ }^{1,2}$, Takuya Araki ${ }^{1,2, *}$, Koujirou \\ Yamamoto ${ }^{1,2}$
1. Department of Clinical Pharmacology and Therapeutics, Gunma University Graduate School of Medicine, Maebashi 371-8511, Japan \\ 2. Department of Pharmacy, Gunma University Hospital, Maebashi 371-8511, Japan
}

Received : 10 Dec 2018/Revised : 30 Dec 2018/Accepted :2 Jan 2019/Published 21 Jan 2019

\begin{abstract}
Anti-epidermal growth factor receptor (EGFR) monoclonal antibodies (Mabs) show high efficacy in about $50 \%$ of colorectal cancer (CRC) patients with wild-type KRAS. However, $<20 \%$ of patients with KRAS wild-type CRC have continued therapeutic effects with these agents, and acquired resistance to treatment has become a serious clinical problem. In this study, to clarify the factors related to acquisition of resistance to cetuximab and establish countermeasures against such acquired resistance, we conducted a comprehensive protein analysis via a proteomics approach using acquired resistance cell lines derived from cetuximab -sensitive CRC cell lines and original cell lines. Cetuximab-acquired resistance cell lines were generated by continuous exposure of SW48 and C99 cell lines to cetuximab. Expression of deoxycytidine kinase (dCK) and zinc finger and BTB domain-containing protein 41 (ZBTB41) increased more than 10fold, and dual specificity protein phosphatase 3 (DUS3) expression decreased by less than 1/10 with acquisition of resistance to cetuximab in both C99 and SW48 cell lines. Because overexpression of dCK is known as a positive indicator of efficacy of nucleoside analogs such as cytarabine or gemcitabine, it is considered that nucleoside analogs activated by dCK may be useful agents in treatment of cancers with acquired cetuximab-resistance. In the future, we need to clarify the usefulness of these drugs for the treatment of cetuximab resistant CRC and to assess the possibility of restoration of cetuximab sensitivity by regulation of ZBTB41 and DUS3 expression.
\end{abstract}

Keywords:cetuximab, colorectal cancer, acquired resistance, protein, dCK, ZBTB41

\section{Introduction}

Cetuximab was launched in the United States and Europe in 2004 as an anti-epidermal growth factor receptor (EGFR) monoclonal antibody (Mab) for treatment of colorectal cancer (CRC) and pharyngeal cancer. Even in Japan, cetuximab has been widely used to treat CRC and pharyngeal cancer, and is considered a drug of choice, especially in the treatment of CRC. A large-scale clinical trial showed that the presence of genetic variations of KRAS is a resistance factor for antiEGFR Mabs including cetuximab and that the response rate to anti-EGFR Mabs in CRC patients with wild-type KRAS was about 50\%(1-3). However, it is reported that $<20 \%$ of patients with KRAS wild-type CRC who experience continued therapeutic effects of anti-EGFR Mabs, and acquired resistance to treatment has become a serious clinical problem(1-3). As a countermeasure to acquired resistance to anti-cancer drugs, factors affecting development of acquired resistance were investigated in several studies, and several factors including the RAS-RAF-mitogen activated protein kinase (MAPK)/Erk kinase (MEK)-MAPK signaling pathway, which is significantly involved in the proliferation of cancer cells, were reported to affect tolerance to cetuximab(1-3). Troiani and colleagues reported that MEK inhibitors showed high growth inhibition efficacy against cells with acquired resistance established by administering cetuximab to mice inoculated with cancer cells or exposing cultured cells to cetuximab, and that concomitant use of cetuximab and a MEK inhibitor showed stronger growth inhibition efficacy(4). In addition, Zhang et al. reported that down-regulation 
of phosphatase and tensin homolog (PTEN) and an increase in phosphorylated protein kinase $\mathrm{B}, \mathrm{Akt}$ were found in acquired cetuximab-resistant cell lines generated from cetuximab-sensitive cell lines by exposing exosomes extracted from cetuximabresistant cell lines and acquired resistance to cetuximab was canceled by use of Akt inhibitor (5). However, those studies focused were on specific pathways or proteins and could not sufficiently account for factors affecting acquisition of resistance to cetuximab, and thus countermeasures against acquired resistance have not yet been established. To establish appropriate countermeasures against acquired resistance to treatment, the influence of an enormous number of proteins, including proteins other than targets of analysis, have to be assessed and each mechanism of resistance development caused by those factors has to be clarified (6).

In recent years, the proteomics approach has attracted attention as a comprehensive method of protein analysis. The proteomics approach was applied in many studies such as in the search for predictive markers of the efficacy of erlotinib in non-small cell lung cancer (7-11) and that of platinum preparations for ovarian cancer (12), and successfully established many predictive markers of drug efficacy and factors affecting drug susceptibility.

In this study, to clarify the factors related to acquisition of resistance to cetuximab and establish countermeasures against this acquired resistance, we carried out a comprehensive protein analysis via a proteomic approach using acquired resistance cell lines generated from cetuximab-sensitive CRC cell lines and original cell lines.

\section{Method}

\subsection{Materials}

Cetuximab sensitive CRC cell lines, C99 and SW48 without genetic mutations affecting sensitivity to anti-EGFR mAbs, such as KRAS, NRAS, BRAF, and phosphatidylinositol-4,5bisphosphate 3-kinase catalytic subunit alpha (PIK3CA) mutations, and PTEN overexpression, were purchased from the European Collection of
Cell Cultures (Salisbury, UK) and the American Type Culture Collection (Manassas, VA), respectively. Reagents for culture and sample preparation were purchased from Wako Pure Chemical Industries (Osaka, Japan). All other reagents were obtained from commercial sources, and those used to analyze peptides were graded for high-performance liquid chromatography, liquid chromatography-tandem mass spectrometry (LCMS/MS), or analytical use.

\subsection{Cell culture and sample preparation}

The C99 cell line was cultured in a humidified incubator at $37^{\circ} \mathrm{C}$ in the presence of $5 \% \mathrm{CO} 2$, and the SW48 cell line was cultured in a $37^{\circ} \mathrm{C}$ incubator with no supplemental $\mathrm{CO} 2$. Cetuximab-acquired resistance cell lines (SW48-CR and C99-CR) were generated upon continuous exposure of SW48 and C99 cell lines to cetuximab according to the method described by Troiani et al (13). Briefly, SW48 and C99 cell lines were continuously exposed to cetuximab, at a starting concentration of $0.1 \mu \mathrm{g} / \mathrm{mL}$, increased 2-fold every 30 days, to increase the inhibition of $50 \%$ of cancer cell growth (IC50), up to a final concentration of $12.8 \mu \mathrm{g} / \mathrm{mL}$. Cytoplasmic proteins were extracted from $80 \%$ confluent cell lines using a Minute Plasma Membrane Protein Isolation Kit (Invent Biotechnologies, Inc., Plymouth, MN), and the concentrations of the extracted proteins were measured using a DC ${ }^{\mathrm{TM}}$ Protein Assay Kit (BioRad Laboratories, Inc., Hercules, CA). Cytoplasmic protein extracts were diluted to 0.7 $\mathrm{mg} / \mathrm{mL}$, and $180 \mu \mathrm{L}$ of the samples were mixed with $20 \mu \mathrm{L}$ of $5 \mathrm{mg} / \mathrm{mL}$ bovine serum albumin and incubated at $37^{\circ} \mathrm{C}$ for $90 \mathrm{~min}$ with $163 \mathrm{mg}$ urea and $15.4 \mu \mathrm{L}$ of $40 \mathrm{mg} / \mathrm{mL}$ dithiothreitol in $8 \mathrm{~mol} / \mathrm{L}$ urea/0.5 mol/L Tris $\mathrm{HCl}(\mathrm{pH} 8.5)$ for reduction of disulfide bonds. Reduced samples were alkylated by reacting with $38.4 \mu \mathrm{L}$ of $40 \quad \mathrm{mg} / \mathrm{mL}$ iodoacetamide in $8 \mathrm{~mol} / \mathrm{L}$ urea $/ 0.5 \mathrm{~mol} / \mathrm{L}$ Tris- $\mathrm{HCl}$ (pH 8.5) for $30 \mathrm{~min}$ at $37^{\circ} \mathrm{C}$. Subsequently, to digest the proteins, $5.2 \mu \mathrm{L}$ of $1 \mathrm{mg} / \mathrm{mL}$ trypsin in 20 $\mathrm{mmol} / \mathrm{L}$ acetic acid was added to $984 \mu \mathrm{L}$ of samples diluted 4-fold with Milli-Q water, respectively, and trypsinization was performed at $37^{\circ} \mathrm{C}$ overnight. Trypsinized samples were desalted using a 
MonoSpin C18 column (GL Sciences, Inc., Tokyo, Japan).

\subsection{MS analysis}

Liquid chromatography/mass spectrometry (LC/MS) analysis was performed with the EksigentNanoLC 425 coupled to Triple TOF 6600 (AB Sciex, Tokyo, Japan) interfaced to a NanoSpray III ion source. First, $10 \mu \mathrm{L}$ of the samples were loaded onto a trap column (Acclaim PepMap $100 \mathrm{C} 18,5 \mu \mathrm{m}, 0.2 \mathrm{~mm}$ I.D. $\times 10 \mathrm{~mm}$, Thermo Fischer Scientific K.K., Tokyo, Japan), and were then separated using an analytical column (Acclaim PepMap 100 C18, $3 \mu \mathrm{m}, 0.075$ mm I.D. $\times 250 \mathrm{~mm}$, Thermo Fischer Scientific) with a gradient from 2 to $32 \%$ solvent $\mathrm{B}$ at a flow rate 300 $\mathrm{nL} / \mathrm{min}$ for $120 \mathrm{~min}$ (solvent $\mathrm{A}: 0.1 \%$ formic acid in water; solvent B: $0.1 \%$ formic acid in acetonitrile). Ion source parameters were set as follows: ion source voltage (ISVF) $2350 \mathrm{~V}$, ion source gas (GS1 and GS2) 5 and 0, interface heater temperature $150 \mathrm{C}$, declustering potential (DP) $80 \mathrm{~V}$.

2.4. Sequential Window Acquisition of All Theoretical Mass Spectra (SWATH) data analysis

SWATH runs were acquired using the 100 SWATH variable window method (AB Sciex Pte. Ltd., Singapore) from $\mathrm{m} / \mathrm{z} 100$ to 1800 with each 25 ms accumulation time. Library samples were prepared by mixing samples obtained from 4 cell lines (C99, C99-CR, SW48, SW48-CR) equally, and measured 6 times by data dependent acquisition (DDA) selecting the top 25 highest peaks found in the survey scan. Database searching for the library was performed with ProteinPilot ver. 5.0 (AB Sciex) using the database downloaded from Uniprot (uniprot_sprot.fasta, released 23 Nov. 2017). The 6 DDA runs were combined and analyzed using the Paragon algorithm. The resulting identified proteins with global false discovery rate (FDR) 1\% (1596 proteins) were imported into SWATH Acquisition MicroApp ver. 2.0 (AB Sciex) on Peak View ver. 2.2 (AB Sciex) as the library. The extracted ion chromatograms of
12 SWATH runs were mined for 5 transitions per peptide and 5 peptides per protein, and then processed with a peptide confidence threshold of $99 \%$ and a false discovery rate $<1 \%$.

\subsection{Data analysis}

Data on expression levels of proteins analyzed by SWATH was adjusted by peak intensity of human serum albumin. Proteins for which expression levels in acquired resistance cell lines changed more than 2- or 0.5 -fold compared to original cell lines along with acquisition of resistance were used as specific proteins in cetuximab-resistant or cetuximab-sensitive cell lines. Gene ontology (GO) analysis was performed to analyze relevance of proteins specific to cetuximab-resistant cell lines or -sensitive cell lines was analyzed using The Database for Annotation, Visualization and Integrated Discovery (DAVID) v6. 8 (https://david.ncifcrf.gov).

\section{Result}

SWATH analysis detected 4,179 kinds of peptides composed of 20,895 peaks; 1,294 kinds of proteins were identified and quantified.

In the C99 cell line, the expression levels of 95 proteins increased more than two-fold with acquisition of resistance, and 3 of 33 proteins whose expression levels increased more than 10fold were glycolysis/gluconeogenesis-related proteins (Figure 1). On the other hand, the expression levels of 230 proteins decreased to half or less, and 3 of 29 proteins whose expression levels decreased to $1 / 10$ or less were spliceosomerelated proteins (Figure 1). Likewise, in the SW48 cell line, the expression levels of 91 proteins increased more than double with acquisition of resistance, and the expression level increased more than 10 -fold in 30 kinds of proteins, but no relationship was found among them. The expression levels of 147 proteins decreased to half or less and the expression levels of 20 kinds of proteins decreased to $1 / 10$ or less (Figure 2), but no relationship was found for each protein. 


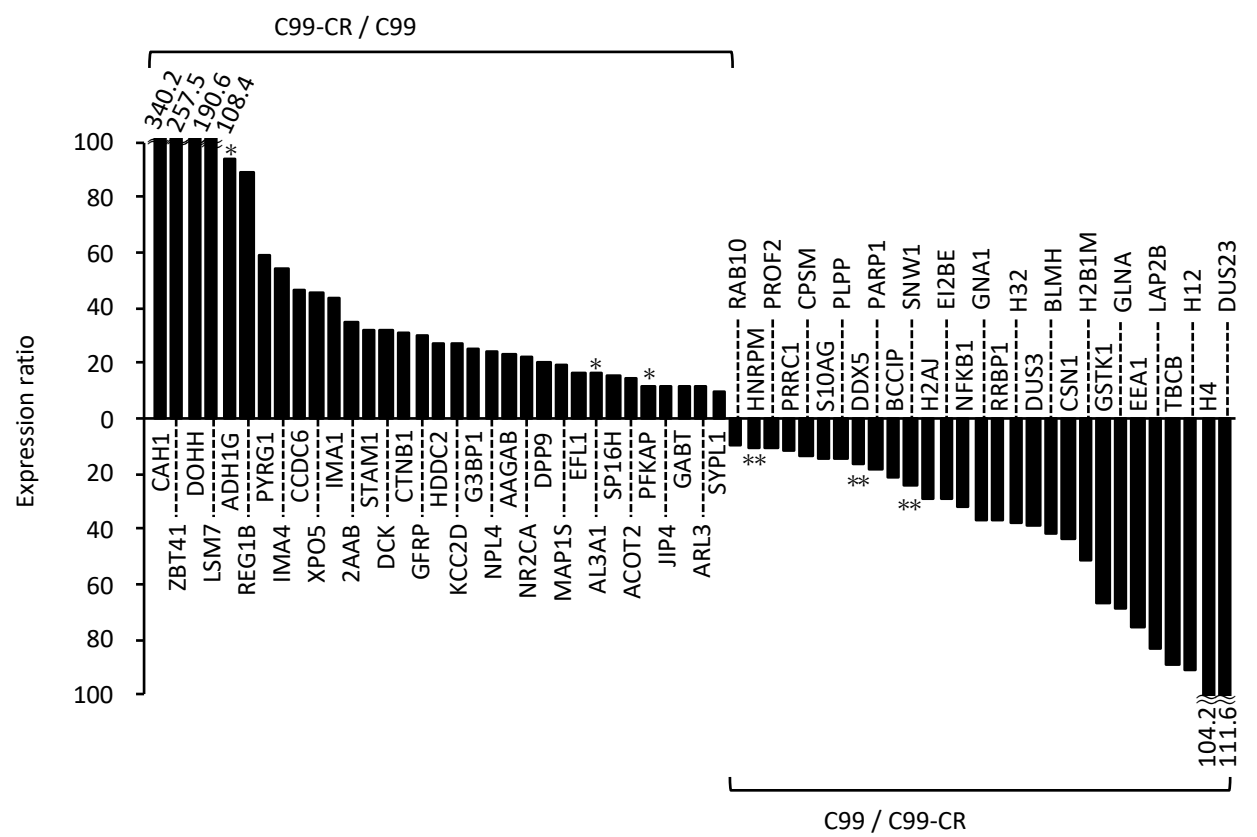

Figure 1. Proteins whose expression level increased 2-fold or decreased to less than half with acquisition of resistance to cetuximabin the $\mathrm{C} 99$ cell line $\left(*\right.$ and ${ }^{* *}$ denote glycolysis/gluconeogenesis related proteins and spliceosome related proteins, respectively)

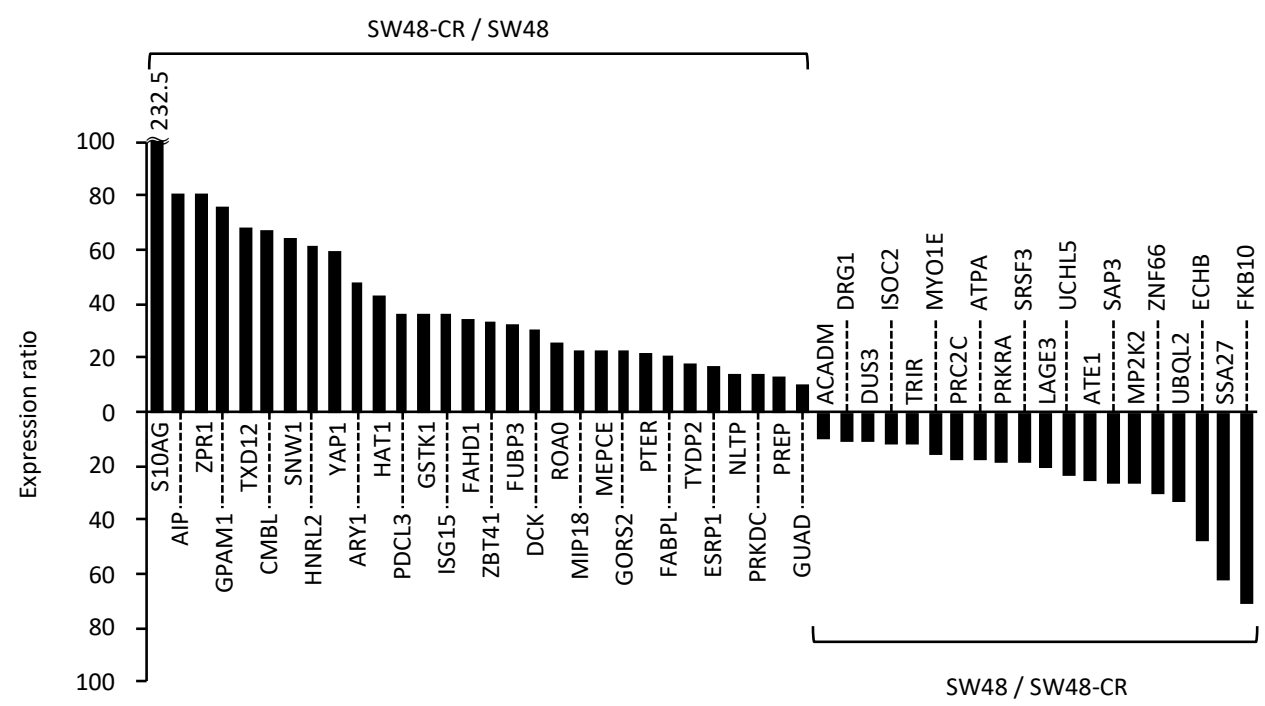

Figure 2. Proteins whose expression level increased more than 2 -fold or deceased to less than half with acquisition of resistance to cetuximabin the SW48 cell line. 


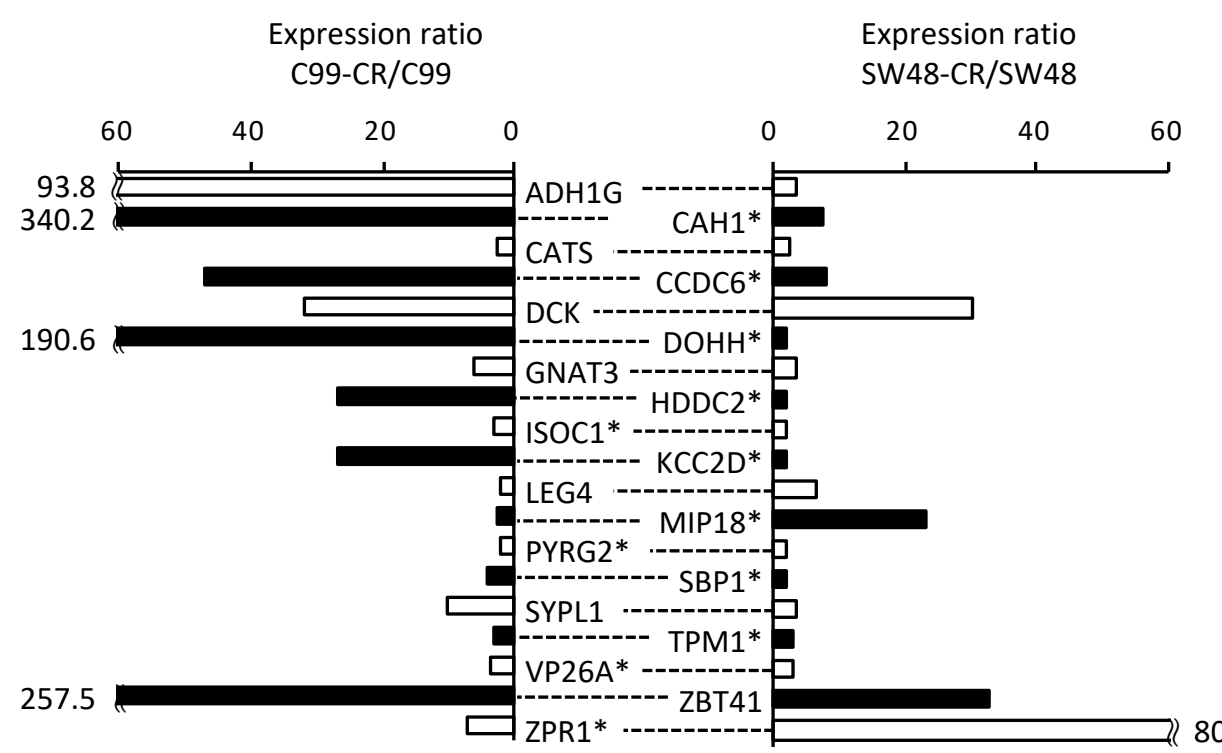

Figure 3. Proteins whose expression level increased more than 2 -fold with acquisition of resistance to cetuximabin both C99 and SW48 cell lines (* denotes proteins with "molecular function" of "protein binding")

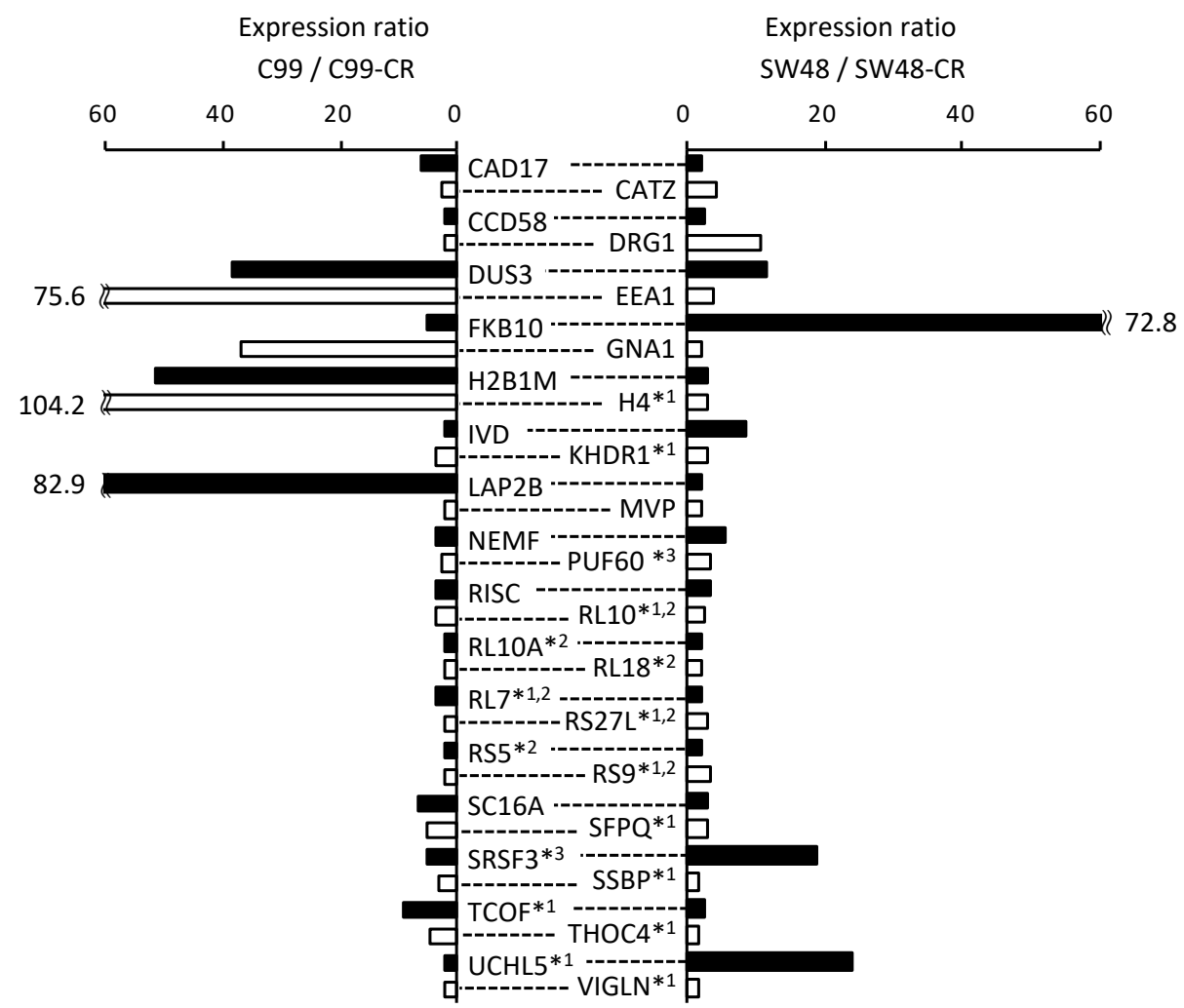

Figure 4. Proteins whose expression level decreased to less than half with acquisition of resistance to cetuximabin both C99 and SW48 cell lines (*1, denotes proteins with "molecular functions" of "poly(A) RNA binding" and "cellular component" of "nucleus", *2 denotes ribosomal proteins, and *3 denotes spliceosome proteins, respectively.

As proteins which increased more than doubled with acquisition of resistance to cetuximabin both of C99 and SW48 cell lines, 19 kinds of proteins were found. GO analysis showed that 12 of 19 proteins have "protein binding as a molecular function" as a common factor, but no relationship 
was found among these proteins (Figure 3). Deoxycytidine kinase (dCK) and zinc finger and BTB domain-containing protein 41 (ZBTB41) increased more than 10 -fold after acquiring resistance in both of C99 and SW48. On the other hand, the expression levels of 32 proteins decreased to half or less in both of C99 and SW48 with acquisition of resistance to cetuximab(Figure 4). GO analysis showed that 12 of 32 proteins have "nucleus as a cellular component" and "poly (A) RNA binding as a common factor" as common factors. In addition, 7 of 32 proteins were constituent proteins of ribosomes and other 3 proteins were constituent proteins of spliceosomes. Dual specificity protein phosphatase 3 (DUS3) decreased to less than $1 / 10$ after acquiring resistance in both of C99 and SW48 (Figure 4).

\section{Discussion}

The proteins obtained from cetuximabresistance cell lines generated by exposing cetuximabsensitive cell lines to cetuximaband that from original cell lines were analyzed, and increase of $\mathrm{dCK}$ and ZBTB41 and decrease of DUS3 were found as a common factor related to acquisition of resistance to cetuximabin both C99 and SW48 cell lines.

$\mathrm{dCK}$ is an enzyme catalyzing the phosphate esterification reaction of the 5'-OH of deoxynucleosides, which is the rate-determining step in the nucleoside salvage pathway. In cancer chemotherapy, dCK is an enzyme needed for phosphorylation of several deoxyribonucleosides and their nucleoside analogs such as gemcitabine and cytarabine and is known as a rate limiting enzyme in activation of these drugs. Furthermore, in addition to a report that the deficiency of dCK is involved in resistance to gemcitabine and cytarabine, it has also been reported that these drugs show very high anti-tumor efficacy in cancer cells that overexpress dCK(14-17). We found for the first time that the expression levels of dCK increase with the acquisition of resistance to cetuximabin this study. Although the reasons and mechanisms for activation of the metabolic pathway of nucleic acids regarding acquisition of resistance to cetuximabinhibiting EGFR pathway is unknown, it is considered that the pathway with the activation of $\mathrm{dCK}$ as the final reaction may be activated as an alternative pathway to the EGFR pathway. In addition, although more detailed study using more cetuximab-resistant cell lines is needed, nucleoside analogs activated by $\mathrm{dCK}$, such as gemcitabine or cytarabine, may be also be useful agents for treating cancers that have acquired tolerance to cetuximab.

On the other hand, the impact of increased ZBTB41 expression and decreased DUS3 expression on the efficacy of chemotherapy has not been reported. ZBTB41 is a protein involved in the stabilization of various proteins such as ribosomal proteins and is involved in the regulation of expression of various proteins (18), and overexpression of ZBTB41 is known to be a poor prognostic factor in liver cancer (19). DUS3 is a member of the dual-specificity protein phosphatase subfamily. By dephosphorylation of phosphorylated residues, DUS3 negatively regulates certain pathways, such as the MAPK pathway, which are associated with cellular proliferation and differentiation (20). Although both of ZTBT41 and DUS3 regulate several protein expression levels, the impact of fluctuation of those proteins on the efficacy of chemotherapy has not been studied. In the future, it is necessary to clarify the significance of variations in ZBTB41 and DUS3 expression levels by analyzing the change in expression levels of several proteins along with changes in ZBTB41 and DUS3 expression levels and assess the possibility of restoration of cetuximab sensitivity by regulation of ZBTB41 and DUS3 expression.

In addition, glycolysis/gluconeogenesis-related proteins were detected as a cetuximab resistancerelated factor in C99 cells. The relationship between drug resistance and changes in expression of glycolysis/gluconeogenesis-related proteins has been reported in several papers, and cancer cells are considered to produce energy as required for survival in the microenvironment by altering the energy production pathway. However, because an increase in glycolysis/gluconeogenesis-related proteins was observed in only C99 but not in SW48 cells, the significance of this change varies greatly depending on the cell line, and thus it would be 
difficult to establish a therapeutic target for cetuximab-resistant tumors.

In this study, because proteomic assay was performed using whole cells, we could not evaluate proteins with low expression such as EGFR pathway-related proteins, which is the underlying pathway in the mechanism of action of cetuximab. To evaluate the factors affecting acquisition of resistance to cetuximab, more detailed study focusing on proteins with low expression is needed.

\section{Conclusion}

In conclusion, we have revealed for the first time a change in dCK, ZBTB41, and DUS3 expression levels, largely with acquisition of resistance to cetuximab. Our data suggest that nucleoside analogs such as cytarabine and gemcitabine may be useful for treating CRC with acquired resistance to cetuximab. In future, it would be necessary elucidate the usefulness of these drugs for the treatment of cetuximab-resistant CRC and to assess the possibility of restoration of cetuximab sensitivity by regulation of ZBTB41 and DUS3 expression.

\section{Acknowledgements}

We thank Dr. Touko Hirano (Laboratory for Analytical Instruments, Education and Research Support Center, Gunma University Graduate School of Medicine) and Dr. Mitsue Miyazaki (Division of Endocrinology, Metabolism and Signal Research, Gunma University Initiative for Advanced Research) for excellent technical assistance. This work was supported by JSPS KAKENHI Grant Number JP16H00504 and JP18K06743.

\section{References}

[1]Karapetis CS, Khambata-Ford S, Jonker DJ, O'Callaghan CJ, Tu D, Tebbutt NC, et al. K-ras Mutations and Benefit from Cetuximab in Advanced Colorectal Cancer. N Engl J Med. 2008;359(17):1757-65.

[2]Therkildsen C, Bergmann TK, HenrichsenSchnack T, Ladelund S, Nilbert $M$. The predictive value of KRAS, NRAS, BRAF, PIK3CA and PTEN for anti-EGFR treatment in metastatic colorectal cancer: A systematic review and meta-analysis. Acta Oncol. 2014;53(7):852-64.

[3]Xu JM, Wang Y, Wang YL, Wang Y, Liu T, Ni $\mathrm{M}$, et al. PIK3CA Mutations Contribute to Acquired Cetuximab Resistance in Patients with Metastatic Colorectal Cancer. Clin Cancer Res. 2017;23(16):4602-4616.

[4]Troiani T, Napolitano S, Vitagliano D, Morgillo F, Capasso A, Sforza V, et al. Primary and Acquired Resistance of Colorectal Cancer Cells to Anti-EGFR Antibodies Converge on MEK/ERK Pathway Activation and Can Be Overcome by Combined MEK/EGFR Inhibition. Clin Cancer res. 2014;20(14):377586.

[5]Zhang S, Zhang Y, Qu J, Che X, Fan Y, Hou K, et al. Exosomes promote cetuximab resistance via the PTEN/Akt pathway in colon cancer cells. Braz J Med Biol Res. 2018;51(1):e6472.

[6]Zhang B, Wang J, Wang X, Zhu J, Liu Q, Shi Z, et al. Proteogenomic characterization of human colon and rectal cancer. Nature. 2014;513(7518):382-7.

[7]Chung CH, Seeley EH, Roder H, Grigorieva J, Tsypin M, Roder J, et al. Detection of tumor epidermal growth factor receptor pathway dependence by serum mass spectrometry in cancer patients. Cancer Epidemiol Biomarkers Prev. 2010;19(2):358-65.

[8]Pitteri SJ, Amon LM, BusaldBuson T, Zhang Y, Johnson MM, Chin A, et al. Detection of elevated plasma levels of epidermal growth factor receptor before breast cancer diagnosis among hormone therapy users. Cancer Res. 2010;70(21):8598-606.

[9]Garrisi VM, Bongarzone I, Mangia A, Cremona M, De Bortoli M, Vaghi E, et al. Characterization of a serum protein pattern from NSCLC patients treated with Gefitinib. ClinBiochem. 2011;44(10-11):936-40.

[10]Lazzari C, Spreafico A, Bachi A, Roder H, Floriani I, Garavaglia D, et al. Changes in plasma mass-spectral profile in course of treatment of non-small cell lung cancer patients with epidermal growth factor receptor tyrosine kinase inhibitors. J Thorac Oncol. 2012;7(1):408. 
[11] Gregorc V, Novello S, Lazzari C, Barni S, Aieta M, Mencoboni M, et al. Predictive value of a proteomic signature in patients with nonsmall-cell lung cancer treated with second-line erlotinib or chemotherapy (PROSE): a biomarker-stratified, randomised phase 3 trial. Lancet Oncol. 2014;15(7):713-21.

[12] Yu KH, Levine DA, Zhang H, Chan DW, Zhang Z, Snyder M. Predicting Ovarian Cancer Patients' Clinical Response to Platinum-Based Chemotherapy by Their Tumor Proteomic Signatures. J Proteome Res. 2016;15(8):245565.

[13] Troiani T, Martinelli E, Napolitano S, Vitagliano D, Ciuffreda LP, Costantino S, et al. Increased TGF- $\alpha$ as a mechanism of acquired resistance to the anti-EGFR inhibitor cetuximab through EGFR-MET interaction and activation of MET signaling in colon cancer cells. Clin Cancer Res. 2013;19(24):6751-65.

[14]Hapke DM, Stegmann AP, Mitchell BS. Retroviral transfer of deoxycytidine kinase into tumor cell lines enhances nucleoside toxicity. Cancer Res. 1996;56(10):2343-7.

[15] Blackstock AW, Lightfoot H, Case LD, Tepper JE, Mukherji SK, Mitchell BS, et al. Tumor uptake and elimination of 2',2'-difluoro-2'deoxycytidine (gemcitabine) after deoxycytidine kinase gene transfer: correlation with in vivo tumor response. Clin Cancer Res. 2001;7(10):3263-8.

[16] Kurata M, Rathe SK, Bailey NJ, Aumann NK, Jones JM, Veldhuijzen GW, et al. Using genome-wide CRISPR library screening with library resistant DCK to find new sources of Ara-C drug resistance in AML. Sci Rep. 2016;6:36199.

[17] Woo SM, Yoon KA, Hong EK, Park WS, Han SS, Park SJ, et al. DCK expression, a potential predictive biomarker in the adjuvant gemcitabine chemotherapy for biliary tract cancer after surgical resection: results from a phase II study. Oncotarget. 2017;8(46):8139481404.

[18]Zhang H, Morrison MA, Dewan A, Adams S, Andreoli M, Huynh N, et al. The NEI/NCBI dbGAP database: genotypes and haplotypes that may specifically predispose to risk of neovascular age-related macular degeneration. BMC Med Genet. 2008;9(51).

[19]Zhou L, Du Y, Kong L, Zhang X, Chen Q. Identification of molecular target genes and key pathways in hepatocellular carcinoma by bioinformatics analysis. Onco Targets Ther. 2018;11:1861-1869.

[20]Russo LC, Farias JO, Ferruzo PYM, Monteiro LF, Forti FL. Revisiting the roles of VHR/DUSP3 phosphatase in human diseases. Clinics (Sao Paulo). 2018;73(suppl 1):e466s. 